Journal of Physics and Its Applications

Journal homepage : https://ejournal2.undip.ac.id/index.php/jpa/index

\title{
Some Studies on Lorentz Transformation Matrix in Non-Cartesian Co-ordinate System
}

\author{
Mukul Chandra Das ${ }^{1}$, Rampada Misra ${ }^{2}$ \\ 1Satmile High School, Satmile, Contai, West Bengal, India, E- mail: mukuldas.100@gmail.com \\ ${ }_{2}$ Ex-Reader, Department of Physics, P.K.College, Contai, West Bengal, India
}

\section{A R T I C L E I N F O}

Article history:

Received: 21 May 2019

Accepted: 13 June 2019

Available online: 15 June 2019

\section{Keywords:}

Lorentz transformation,

frame of reference,

system of co-ordinates

\begin{abstract}
A B S T R A C T
The Lorentz matrices for transformation of co-ordinates in Cartesian system are presented for the cases when the relative velocity between two reference frames is along $X, Y$ and $Z$ axes. The general form of the matrix for transformation of co-ordinates from unprimed to primed frame has been deduced in case of Cartesian co-ordinate system with the help of the above matrices. This matrix has not been transformed to the cases of cylindrical and spherical polar co-ordinates due to the fact that the calculations are cumbersome and lengthy. Hence, considering the relative velocity between two frames along a co-ordinate axis the transformation matrix has been found out for cylindrical and spherical co-ordinates.
\end{abstract}

\section{Introduction}

Our knowledge of special theory of relativity tells us that Lorentz transformation matrix is a mathematical tool for transforming co-ordinates, velocities and many other characteristics of matter in Cartesian co-ordinate system when there is a relative velocity between two frames of references. In literature it is found that several workers have put forward the matrix for Lorentz transformation of coordinates in cases of $3 D$ and $4 D$ spaces [1-3]. The velocity of matter was, also, dealt with by some other workers [4]. In our earlier works Lorentz transformation was, also, dealt with considering linear motion, rotation etc. of frame of references $[5,6]$.

It is to be pointed out that all the works, mentioned above, were done on the basis of the transformations in Cartesian co-ordinate system. The elements of the transformation matrix would, evidently, change if cylindrical or spherical polar coordinates be considered. It could be shown that the calculations for general form of the transformation matrix in cylindrical and spherical co-ordinates are lengthy and cumbersome. So, we set aside the calculation of the general form of these transformation matrices for the present. In this work the general form of the Lorentz matrix for coordinate transformation in Cartesian system would be found out taking the relative velocity of a frame of reference with respect to another in any arbitrary direction. We, also, proceed to find out the transformation matrix for cylindrical and spherical systems of co-ordinates when the velocity of $S^{\prime}$ is along any one of the axes of co-ordinates of $S$. For the present, let us take the case of motion of $S^{\prime}$ along
$X$ axis of $S$. For other two axes the procedure would be similar.

\section{Lorentz transformation for relative velocity in any arbitrary direction:}

We know that when a frame $S^{\prime}$ moves linearly with velocity $v$ along positive direction of $X$-axis with respect to a frame $S$ then, in Cartesian co-ordinate system, Lorentz transformation of co-ordinates in $S$ to those in $S^{\prime}$ would be given by $[7,8,9]$

$$
x_{i}^{\prime}=a_{i j} x_{j}
$$

where $a_{i j}$ is the Lorentz transformation matrix of rank two whereas $x_{i}^{\prime}$ and $x_{j}$ are tensors of rank one. Equation (1) is applicable to 4-vector transformation.

Let the frame $S^{\prime}$ moves linearly at velocity $v$ with respect to the frame $S$ along any arbitrary direction inclined to the co-ordinate axes. One can consider that $S^{\prime}$ has simultaneous rectilinear motions with velocities $v_{x}, v_{y}$ and $v_{z}$ respectively along $X, Y$ and $Z$-axes. We can think about the situation as follows. This means that $v_{x}, v_{y}$ and $v_{z}$ are the components of $v$ along $X, Y$ and $Z$-axes respectively.

Let there be four reference frames $S^{\prime}, \overline{\bar{S}}, \bar{S}$ and $S$. We suppose that $S^{\prime}$ is in $\overline{\bar{S}}, \overline{\bar{S}}$ is contained in $\bar{S}$ and $\bar{S}$ is within $S$. Here, $\bar{S}$ moves with velocity $v_{x}$ 
along a direction parallel to $X$-axis, $\overline{\bar{S}}$ moves with $v_{y}$ parallel to $\bar{Y}$-axis and $S^{\prime}$ moves with $v_{z}$ aligned parallel to $\overline{\bar{Z}}$-axis. There is no rotation of the frames. Therefore, Lorentz transformation of co-ordinates from $S$ to $\bar{S}$, from $\bar{S}$ to $\overline{\bar{S}}$ and from $\overline{\bar{S}}$ to $S^{\prime}$ could be respectively given by

$$
\bar{x}_{r}=A_{r s} x_{s}, \quad \overline{\bar{x}}_{q}=B_{q r} \bar{x}_{r}, \quad x_{p}^{\prime}=C_{p q} \overline{\bar{x}}_{q}
$$

Hence, Lorentz transformation of co-ordinates from $S$ to $S^{\prime}$ could be given by

$$
x_{p}^{\prime}=C_{p q} B_{q r} A_{r s} x_{s}
$$

where $A_{r s}, B_{q r}$ and $C_{p q}$ are respectively the Lorentz transformation matrices for velocities $v_{x}, v_{y}$ and $v_{z}$ along $X, Y$ and $Z$-axes respectively which could be easily written as

$$
A_{r s}=\left(\begin{array}{cccc}
\gamma_{x} & 0 & 0 & -\gamma_{x} v_{x} \\
0 & 1 & 0 & 0 \\
0 & 0 & 1 & 0 \\
-\frac{\gamma_{x} v_{x}}{c^{2}} & 0 & 0 & \gamma_{x}
\end{array}\right)
$$

$$
B_{q r}=\left(\begin{array}{cccc}
1 & 0 & 0 & 0 \\
0 & \gamma_{y} & 0 & -\gamma_{y} v_{y} \\
0 & 0 & 1 & 0 \\
0 & -\frac{\gamma_{y} v_{y}}{c^{2}} & 0 & \gamma_{y}
\end{array}\right)
$$

$$
C_{p q}=\left(\begin{array}{cccc}
1 & 0 & 0 & 0 \\
0 & 1 & 0 & 0 \\
0 & 0 & \gamma_{z} & -\gamma_{z} v_{z} \\
0 & 0 & -\frac{\gamma_{z} v_{z}}{c^{2}} & \gamma_{z}
\end{array}\right)
$$

where $\gamma_{i}=\frac{1}{\sqrt{1-\frac{v_{i}^{2}}{c^{2}}}}, i=x, y, z$. If we write

$$
A_{p s}=C_{p q} B_{q r} A_{r s}
$$

then (3) becomes

$$
x_{p}^{\prime}=A_{p s} x_{s}
$$

The above considerations imply that one can make Lorentz transformation between two frames having relative velocity in any arbitrary direction. Of course,
$A_{p s}$ is in Cartesian co-ordinates only. Using (4), (5), (6) and (7) we obtain

$$
A_{p s}=\left(\begin{array}{cccc}
\gamma_{x} & 0 & 0 & -\gamma_{x} v_{x} \\
\frac{\gamma_{x} \gamma_{y} v_{x} v_{y}}{c^{2}} & \gamma_{y} & 0 & -\gamma_{x} \gamma_{y} v_{y} \\
\frac{\gamma_{x} \gamma_{y} \gamma_{z} v_{x} v_{z}}{c^{2}} & \frac{\gamma_{y} \gamma_{z} v_{y} v_{z}}{c^{2}} & \gamma_{z} & -\gamma_{x} \gamma_{y} \gamma_{z} v_{z} \\
-\frac{\gamma_{x} \gamma_{y} \gamma_{z} v_{x}}{c^{2}} & -\frac{\gamma_{y} \gamma_{z} v_{y}}{c^{2}} & -\frac{\gamma_{z} v_{z}}{c^{2}} & \gamma_{x} \gamma_{y} \gamma_{z}
\end{array}\right)
$$

This matrix may be regarded as the general matrix including the simultaneous motion of $S^{\prime}$ along the three co-ordinate axes with respect to $S$. It could be easily shown that (9) leads to the individual cases cited in (4), (5) and (6) on proper consideration of $v_{x}, v_{y}, v_{z}$ and corresponding values of $\gamma$.

It is to be noted that in equation (8) $A_{p s}$ is the Lorentz transformation matrix in Cartesian coordinate system. According to the principle of tensor we can write

$$
\bar{A}_{\alpha \beta}=\frac{\partial x^{p}}{\partial \bar{x}^{\alpha}} \frac{\partial x^{s}}{\partial \bar{x}^{\beta}} A_{p s}
$$

Here, $\bar{A}_{\alpha \beta}$ would be the general form of co-ordinate transformation matrix in cylindrical or spherical polar co-ordinates if we write $x^{i}$ and $\bar{x}^{j}(i, j=1,2,3,4)$ accordingly.

\section{Case of Cylindrical Co-ordinate System:}

To transfer a matrix in Cartesian co-ordinates to that in cylindrical co-ordinates let us consider a point in $S$ having co-ordinates $(x, y, z, t)$ in Cartesian system and $(\rho, \theta, z, t)$ in cylindrical system of coordinates. Here, $S^{\prime}$ is moving with velocity $v$ along $Z$ with respect to $S$. Let us write

$$
\begin{aligned}
& x^{1}=x, x^{2}=y, x^{3}=z, x^{4}=t ; \\
& \bar{x}^{1}=\rho, \bar{x}^{2}=\rho \theta, \bar{x}^{3}=z, \bar{x}^{4}=t ; \\
& x=\rho \operatorname{Cos} \theta, y=\rho \operatorname{Sin} \theta, z=z, t=t
\end{aligned}
$$

In this case, it is known that $\bar{x}^{1}, \bar{x}^{2}, \bar{x}^{3}, \bar{x}^{4}$ represent the coordinates along $\rho, \theta, \mathrm{z}, t$ direction respectively

Therefore, one can write

$\frac{\partial x^{1}}{\partial \bar{x}^{1}}=\frac{\partial x}{\partial \rho}=\operatorname{Cos} \theta, \frac{\partial x^{1}}{\partial \bar{x}^{2}}=\frac{1}{\rho} \frac{\partial x}{\partial \theta}=-\sin \theta$,

$\frac{\partial x^{1}}{\partial \bar{x}^{3}}=\frac{\partial x}{\partial z}=0, \frac{\partial x^{1}}{\partial \bar{x}^{4}}=\frac{\partial x}{\partial t}=0$, 
$\frac{\partial x^{2}}{\partial \bar{x}^{1}}=\frac{\partial y}{\partial \rho}=\sin \theta, \frac{\partial x^{2}}{\partial \bar{x}^{2}}=\frac{1}{\rho} \frac{\partial y}{\partial \theta}=\cos \theta$,

$\frac{\partial x^{2}}{\partial \bar{x}^{3}}=\frac{\partial y}{\partial z}=0, \frac{\partial x^{2}}{\partial \bar{x}^{4}}=\frac{\partial y}{\partial t}=0$

$\frac{\partial x^{3}}{\partial \bar{x}^{1}}=\frac{\partial z}{\partial \rho}=0, \frac{\partial x^{3}}{\partial \bar{x}^{2}}=\frac{1}{\rho} \frac{\partial z}{\partial \theta}=0$,

$\frac{\partial x^{3}}{\partial \bar{x}^{3}}=\frac{\partial z}{\partial z}=1, \frac{\partial x^{3}}{\partial \bar{x}^{4}}=\frac{\partial z}{\partial t}=0$

$\frac{\partial x^{4}}{\partial \bar{x}^{1}}=\frac{\partial t}{\partial \rho}=0, \frac{\partial x^{4}}{\partial \bar{x}^{2}}=\frac{1}{\rho} \frac{\partial t}{\partial \theta}=0$,

$\frac{\partial x^{4}}{\partial \bar{x}^{3}}=\frac{\partial t}{\partial z}=0, \frac{\partial x^{4}}{\partial \bar{x}^{4}}=\frac{\partial t}{\partial t}=1$

Now, using $\left(C_{p q}\right)$ given in (6) we shall obtain the Lorentz matrix for transformation of co-ordinates from Cartesian to cylindrical system with the help of

$$
\left(\bar{A}_{\alpha \beta}\right)_{c y l}=\frac{\partial x^{r}}{\partial \bar{x}^{\alpha}} \frac{\partial x^{s}}{\partial \bar{x}^{\beta}} A_{r s}
$$

which comes out to be

$$
\left(\bar{A}_{\alpha \beta}\right)_{c y l}=\left(\begin{array}{cccc}
1 & 0 & 0 & 0 \\
0 & 1 & 0 & 0 \\
0 & 0 & \gamma_{z} & -\gamma_{z} v_{z} \\
0 & 0 & -\frac{\gamma_{z} v_{z}}{c^{2}} & \gamma_{z}
\end{array}\right)
$$

This is analogous to the equation (6). Now, one can write

$$
\left(\begin{array}{c}
x^{\prime 1} \\
x^{\prime 2} \\
x^{\prime 3} \\
x^{\prime 4}
\end{array}\right)\left(\begin{array}{cccc}
1 & 0 & 0 & 0 \\
0 & 1 & 0 & 0 \\
0 & 0 & \gamma_{z} & -\gamma_{z} v_{z} \\
0 & 0 & -\frac{\gamma_{z} v_{z}}{c^{2}} & \gamma_{z}
\end{array}\right)\left(\begin{array}{l}
\bar{x}^{1} \\
\bar{x}^{2} \\
\bar{x}^{3} \\
\bar{x}^{4}
\end{array}\right)
$$

This also satisfies the relation

$$
\begin{gathered}
(d \rho)^{2}+\rho^{2}(d \theta)^{2}+(d z)^{2}-c^{2}(d t)^{2}= \\
\left(d \rho^{\prime}\right)^{2}+\rho^{\prime 2}\left(d \theta^{\prime}\right)^{2}+\left(d z^{\prime}\right)^{2}-c^{2}\left(d t^{\prime}\right)^{2}
\end{gathered}
$$

which justifies the above transformation.

4. Case of Spherical Co-ordinate System:

To transfer a matrix in Cartesian system to that in spherical co-ordinate system let us consider a point in $S$ having co-ordinates $(x, y, z, t)$ in Cartesian system and $(r, \theta, \phi, t)$ in spherical system of coordinates. Here, $S^{\prime}$ is moving with velocity $v$ along $r$ with respect to $S$.

Therefore, velocity component along $\mathrm{X}, \mathrm{Y}$ and $\mathrm{Z}$ axis respectively are $v_{x}=v \operatorname{Sin} \theta \operatorname{Cos} \phi, v_{y}=v \operatorname{Sin} \theta \operatorname{Sin} \phi, v_{z}=v \operatorname{Cos} \theta$

In this case, Lorentz transformation matrix in Cartesian form will be as in equation (9).

Now, let us write

$x^{1}=x, x^{2}=y, x^{3}=z, x^{4}=t ;$

$\bar{x}^{1}=r, \bar{x}^{2}=r \theta, \bar{x}^{3}=\phi r \sin \theta, \bar{x}^{4}=t$

$x=r \operatorname{Sin} \theta \operatorname{Cos} \phi, \quad y=r \operatorname{Sin} \theta \operatorname{Sin} \phi$,

$z=r \operatorname{Cos} \theta, \quad t=t$

It is also, well known that $\bar{x}^{1}, \bar{x}^{2}, \bar{x}^{3}, \bar{x}^{4}$ represent the direction along $r, \theta, \phi, t$ respectively

Therefore, one can write

$\frac{\partial x^{1}}{\partial \bar{x}^{1}}=\frac{\partial x}{\partial r}=\sin \theta \operatorname{Cos} \phi, \frac{\partial x^{1}}{\partial \bar{x}^{2}}=\frac{1}{r} \frac{\partial x}{\partial \theta}=\cos \theta \cos \phi$,

$\frac{\partial x^{1}}{\partial \bar{x}^{3}}=\frac{1}{r \sin \theta} \frac{\partial x}{\partial \phi}=-\operatorname{Sin} \phi, \frac{\partial x^{1}}{\partial \bar{x}^{4}}=\frac{\partial x}{\partial t}=0$,

$\frac{\partial x^{2}}{\partial \bar{x}^{1}}=\frac{\partial y}{\partial r}=\sin \theta \sin \phi, \frac{\partial x^{2}}{\partial \bar{x}^{2}}=\frac{1}{r} \frac{\partial y}{\partial \theta}=\cos \theta \sin \phi$,

$\frac{\partial x^{2}}{\partial \bar{x}^{3}}=\frac{1}{r \sin \theta} \frac{\partial y}{\partial \phi}=\cos \phi, \frac{\partial x^{2}}{\partial \bar{x}^{4}}=\frac{\partial y}{\partial t}=0$

$\frac{\partial x^{3}}{\partial \bar{x}^{1}}=\frac{\partial z}{\partial r}=\cos \theta, \frac{\partial x^{3}}{\partial \bar{x}^{2}}=\frac{1}{r} \frac{\partial z}{\partial \theta}=-\sin \theta$,

$\frac{\partial x^{3}}{\partial \bar{x}^{3}}=\frac{1}{r \sin \theta} \frac{\partial z}{\partial \phi}=0, \frac{\partial x^{3}}{\partial \bar{x}^{4}}=\frac{\partial z}{\partial t}=0$

$\frac{\partial x^{4}}{\partial \bar{x}^{1}}=\frac{\partial t}{\partial r}=0, \frac{\partial x^{4}}{\partial \bar{x}^{2}}=\frac{1}{r} \frac{\partial t}{\partial \theta}=0$,

$\frac{\partial x^{4}}{\partial \bar{x}^{3}}=\frac{1}{r \sin \theta} \frac{\partial t}{\partial \phi}=0, \frac{\partial x^{4}}{\partial \bar{x}^{4}}=\frac{\partial t}{\partial t}=1$

Now, using $\left(A_{p s}\right)$ given in (9) and using the above relations easily, one can obtain the Lorentz transformation matrix in spherical coordinate system with the help of equation

$$
\left(\bar{A}_{\alpha \beta}\right)_{s p h l}=\frac{\partial x^{p}}{\partial \bar{x}^{\alpha}} \frac{\partial x^{s}}{\partial \bar{x}^{\beta}} A_{p s}
$$

which gives the Lorentz transformation from frame $S$ to $S^{\prime}$ in spherical coordinate system as given below.

$$
\left(\begin{array}{c}
x^{\prime 1} \\
x^{\prime 2} \\
x^{\prime 3} \\
x^{\prime 4}
\end{array}\right)=\left(\bar{A}_{\alpha \beta}\right)\left(\begin{array}{l}
\bar{x}^{1} \\
\bar{x}^{2} \\
\bar{x}^{3} \\
\bar{x}^{4}
\end{array}\right)
$$

After calculation and its simplification one may prove 


$$
\begin{aligned}
& (d r)^{2}+r^{2}(d \theta)^{2}+r^{2} \sin ^{2} \theta(d \phi)^{2}-c^{2}(d t)^{2}= \\
& \left(d r^{\prime}\right)^{2}+r^{\prime 2}\left(d \theta^{\prime}\right)^{2}+r^{\prime 2} \sin ^{2} \theta^{\prime}\left(d \phi^{\prime}\right)^{2}-c^{2}\left(d t^{\prime}\right)^{2}
\end{aligned}
$$

\section{Conclusion:}

The matrix obtained in (9) is that for transformation of co-ordinates in Cartesian co-ordinate system when the relativistic velocity $v$ is in any arbitrary direction. This is a general form of Lorentz matrix for transformation of co-ordinates. If motion of $S^{\prime}$ be along any co-ordinate axis then we could obtain (4), (5) or (6) from (9) by substituting other two components of $v$ to be zero and corresponding value of $\gamma$ for the case. Again, the transformation matrix (6) for Cartesian co-ordinate system has been transformed to that in cylindrical system of coordinate as given in (13). Similarly, the transformation matrix (9) for Cartesian co-ordinate system may be transformed to that in spherical coordinate system by the relation (16)

It is pointed out that in this transformation, same dimensional space coordinates have been taken essentially to satisfy the relation invariant under Lorentz transformation. This means that $\bar{x}^{1}, \bar{x}^{2}, \bar{x}^{3}, \bar{x}^{4}$ are same dimensional but represent the direction along $r, \theta, \phi, t$ respectively in spherical system and those represent the direction along $\rho, \theta, \mathrm{z}, t$ respectively in cylindrical system.

However, Lorentz transformation is very important and useful to study the several topics in our physics. But it is mainly confined in Cartesian coordinate system. If, this transformation be developed in non Cartesian coordinate system then it may have important applications in physics.

\section{References:}

1. Iyer C., Prabhu G.M., Lorentz Transformations with Arbitrary Line of Motion, European Journal of Physics, 28, 183. (2007)

2. Iyer C., Prabhu G.M., Composition of Two Lorenz Boosts through Spatial and Space-time Rotations, Journal of Physical and Natural Sciences, 1, 1 (2007)

3. Katz R., An Introduction to the Special Theory of Relativity, D. Van Nostrand, Princeton (1964)

4. Rodes J. A., Semon M. D., Relativistic Velocity Space, Wigner Rotation, and Thomas Precession, American Journal of Physics, 72, 943 (2004)

5. Das M. C., Misra R., Fundamental Tensor of Electromagnetic Field in the System of Photon, Annals of the University of Craiova Physics AUC, 28, 73 (2018)

6. Das M. C., Misra R., Harmonic Module of Electron and Its Space-Time Geometry, Journal of physical science, 22, 183 (2017)

7. Rosser W. G. V., An Introduction to the Theory of Relativity, Butterworth, London. (1964)

8. Mickelsson J.,Ohlsson T., Snellman H., Relativity Theory, Royal Institute of Technology, Stockholm. (2005)

9. Gupta B. D., Rajput B. S., Mathematical Physics, Pragati Prakashan, Meerut, India, 799. (1969) 